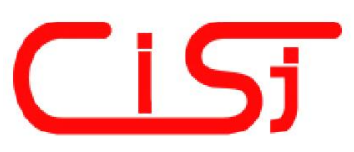

\title{
CELLULAR AUTOMATA MODELING OF IMPURITIES SEGREGATION IN THE MELT CRYSTALLIZATION PROCESS
}

\author{
Liliya Shumylyak ${ }^{1)}$, Vladimir Zhikharevich ${ }^{2)}$ and Sergey Ostapov ${ }^{2)}$ \\ 1) Chernnivtsi Faculty of National Technical University "Kharkiv Politechnical Institute", \\ Chernivtsi, 58012, Ukraine, e-mail: 1shumylyak@mail.ru \\ 2) Yuriy Fedkovych Chernivtsi National University, Chernivtsi, 58012, Ukraine, \\ e-mail: vzhikhar@mail.ru; sergey.ostapov@gmail.com
}

\begin{abstract}
The paper deals with the issue of the construction of a cellular automata model of the directional crystallization of binary solutions process. The basic approach and general methodology for the development of cellular automata models are examined. This allowed to obtain the spatial distribution of the studied characteristics. The paper gives an overview of available techniques on the problem, outlines the arguments in favor of a cellular automata method. The occurring processes of redistribution of impurities concentration and overcooling are emphasized. Previously known idea of a mechanism of the melt concentration overcooling is considered. The results of the calculation of impurity concentration distribution along the track of the sample during crystallization are presented. Dependence of the phase transition melting temperature on the value of the impurity concentration is determined on the basis of the calculated impurity distribution. Graphic examples of the varieties of uneven impurity distribution as a result of overcooling concentration of the melt are given. Copyright (C) Research Institute for Intelligent Computer Systems, 2015. All rights reserved.
\end{abstract}

Keywords: Cellular automata; Phase transition; Segregation; Concentration overcooling.

\section{INTRODUCTION}

It is well known that many physical properties of crystalline materials obtained by the method of directed crystallization are determined by the distribution of impurity in the melt and its ability to accumulate in the form of separate grains, cells etc. This occurs due to concentrated overcooling and leads to deterioration of the mechanical, electrical and physical properties of the material. It is one of the reasons for their fragility. A series of experiments is needed to investigate the optimal conditions for the growth of semiconductor materials with required properties. The time required is not always available, and the labor and material resources cost is rather high. Therefore, in recent years great attention is paid to the development of the technology of process simulation.

Spatial segregation is a complex process to be described in terms of mathematical equations. The imitation or agent-based models where certain rules of conduction can be assigned to each agent are the most popular and flexible techniques at present.

The main difference of cellular automata from ordinary differential equation (DE) lies in the local rules by which the dynamics of the system is described. When using DE we assume the existence of some rules of averaged values over the whole system changes. In the case of the existence of such spacecraft the macro rules are optional. When using the CA the existence of such macro rules is also optional. It is enough to know the laws of the system's development on the microlevel in small spatial regions that make up the macrosystem.

So the main features of a complex dynamic system can be described by simple rules of management, defined from the behavior of the system over time.

CA are the most effectively used to describe the behavior of a system the collective behavior of which is determined by the local behavior of its constituent elements, when the system is highly heterogeneous, and averaging of variables throughout the system can hardly reflect its status adequately as a whole. Therefore, while modeling the melting process, accompanied by the first order phase transition, we chose the cellular automata technique.

Cellular automata were invented by von Neumann [1]; this proved the existence of a selfreproducing universal computer. 
Then the idea became popular and many researchers are working on its development. So several quality classes of cellular automata (CA) behavior, based on various statistical measures are defined in [2]. It was investigated how the cellular automaton is changed by varying entries in its rule table. These abrupt changes have either the character of bifurcations in smooth dynamical systems or that of phase transitions in statistical mechanical systems. Cellular automata classification is difficult. Wolfram classification scheme was developed, consisting of four quality classes, for which all cellular automata can be divided according to the type of evolution [3]. He suggests reliable arguments that the achievements in the field of cellular automata are not isolated but very stable and are of great importance for all areas of science. Such definitions are mostly qualitative and can be interpreted in different ways. Other approaches to CA rules classification were detailed in the works by Chatee and Manneville [4], Gutowitz [5], McIntosh [6].

However, the classification alone is not enough. It is necessary to gain deeper understanding of the nature of the cellular automata interaction rules.

Despite a long period of research of cellular automata, the general theory of cellular automata has not yet been formed. But at the same time, the cellular automata are very successfully used to model various dynamic systems characterized by close interaction between the constituent elements, and this direction proves to be very promising. In recent years, the alternative approaches to numerical methods for problems of heat conductivity and diffusion are widely used. Cellular automata algorithms are quite successfully used $[7,8]$ for this purpose. It should be noted that discrete models are used in most cases for calculation of diffusion processes [9], while the continuous models of cellular automata $[10,11]$ are used to approximate heat transfer processes. In [12] Juan Miguel Benito and Penélope Hernández prove that cellular automata technique is a suitable tool for modeling multi-interactive procedures. Specifically, they used arguments to confirm the simulation results obtained for the classical model of segregation Thomas Schelling [13].

[14] should also be mentioned here in regards to the subject of phase formation modeling using cellular automata. It shows the results of simulation of grain boundary motion when driven by the minimization of volume-stored energy as well as when it is curvature-driven. It also gives an example of a hybrid model that combines cellular automata with the description of diffusion computing and dissolving of precipitation under abnormal grain growth. Furthermore, [15] should be taken into consideration where calculations of austenite-ferrite phase transformation in steels are performed using cellular automata model. Researchers describe the rules of transition for the initial and subsequent growth taking into account internal variables for each CA cell. A qualitative model of the phase transition within the developed cellular automata was presented and sensitivity analysis of the developed complex microscale austenite to ferrite phase transformation model was performed. The possibility of describing complex phenomena and processes using cellular automata enables to simulate not only the phase transition during the crystallization process, but also to sophisticate such a model by the presence of emerging concentration overcooling, which is not modelled in previous works.

In this research we use the method of continuous cellular automata. As it was previously shown in [16], it is possible to display not only the qualitative, but also the quantitative aspect of the modelled process by calculating the time of a CA interaction. This makes it possible to determine the characteristics of the process at certain points of time. Due to its simplicity and versatility, this method is a good alternative to the previously known classical methods for solving problems of heat conductivity, diffusion of impurities, modeling of phase transitions. The description and results of application of continuous cellular automata method for modeling the directional crystallization of binary solutions in dynamics are presented in this paper. Here the segregation and dependence of the phase transition temperature on the material composition is taken into account, because under certain conditions it can lead to the phenomenon of concentration overcooling and, accordingly, to uneven geometry of the crystallization front.

\section{PRELIMINARIES}

It is known that solubility of impurity components in the liquid phase and equilibrium solid phase of the basic substance are different. This is due to different values of the chemical potentials of impurities in the solid and liquid phases.

The difference between the ratio of components in liquid and its equilibrium solid phase is characterized by the distribution coefficient [17]:

$$
K_{0}=C_{S} / C_{L},
$$

where $C_{S}, C_{L}$ - concentration of impurities in the solid (Solidus) and liquid (Liquidus) phases respectively.

The equilibrium distribution coefficient (sometimes referred to as the coefficient of 
segregation or liquidation coefficient) is determined by the type of phase diagram of the corresponding system. When analysing a fragment of the diagram of a two-component system with full mutual solubility at low concentrations of the second component (Fig. 1), it can be noted that the impurity (B) which lowers the melting point of the basic substance (A) will dissolve in the melt better than in the solid phase, i.e. $C_{S}<C_{L}$ and $K_{0}<1$. The crystallized phase will be cleaner than the melt.

If the impurity increases the melting point of the basic substance, it dissolves better in the solid phase than in the melt, i.e. $C_{S}>C_{L}$ and $K_{0}>1$.

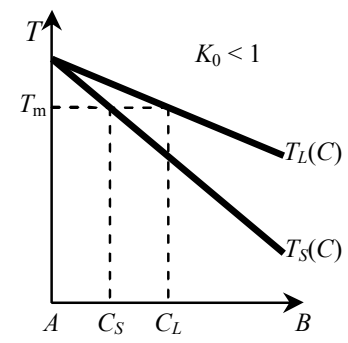

a)

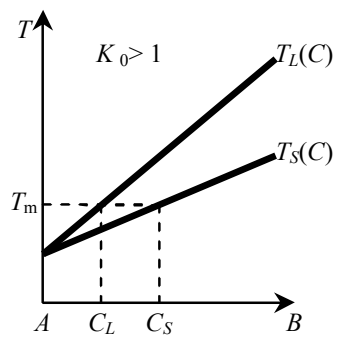

б)
Fig. 1 - Fragments of the diagram of the "basic substance (A) - impurity (B)" state in the area of complete mutual solubility at low concentrations of impurities: a) impurity lowers the melting point; b) impurity increases the melting point of the solution.

The distribution coefficient can be calculated as the ratio of horizontal line segments from the axis of temperature to their crossing with the liquidus and solidus lines.

It should be noted that the equilibrium distribution coefficient is a thermodynamic term that characterizes the process of equilibrium phase transitions. The latter involve transitions from liquid to solid state and back at infinitely low speed or in infinitely small volume.

Since in practice there is a finite speed of the process of phase transition, the concept of effective distribution coefficient $K_{\text {ef }}$ is introduced. It differs from the equilibrium one. The difference between the effective and the equilibrium distribution coefficients arises from the fact that at finite speed of crystallization (system is not in an equilibrium state) the moving front pushes the impurity (if $\left.K_{0}<1\right)$ faster than it can diffuse deep into the melt. Therefore, an impurity-enriched layer, called a diffusion layer, emerges in front of the crystallization front. This layer is characterized by a certain thickness $\delta$. The increase of impurity concentration in the melt in crystallization front leads to its growth in the solid phase. However it is considered that the ratio of impurity concentration in the solid phase to concentration in the liquid phase at the very border of crystallization falls under the equation (1).

The connection between the effective and the equilibrium distribution coefficients is defined by the formula referred to as Burton-Prim-Slichter formula [18]:

$$
K_{\text {ef }}=\frac{K_{0}}{K_{0}+\left(1-K_{0}\right) \exp \left(-\frac{V \delta}{D_{L}}\right)},
$$

where $V$ - speed of crystallization front movement; $D_{L}$ - coefficient of diffusion of impurities in the liquid phase; $\delta$ - thickness of the diffusion layer. Thickness of the diffusion layer depends on both the speed of front movement and the diffusion coefficient of impurities. There may also be a convective or forced mixing of the melt, the intensity of which significantly affects $\delta$.

\section{DESCRIPTION OF A CELLULAR AUTOMATA MODEL}

The essence of the modeling processes of heat conduction via cellular automata is as follows. We divide the sample into sets of identical cells, interconnected in the same way. All cells form the so-called cellular automata lattices. The lattices may be of different dimensions (one-, two- or threedimensional array), depending on the dimensions of the modelled system.

In the case of simulation of complex phenomena involving phase transitions or other transformations, the contents of cells cellular automata field can be of a single linear array for some characteristics.

Let's try to describe the structure of a cellular automata field for our model. The dimension of the field corresponds to the dimension of a simulated system and contains three layers (Fig. 2): 1) cells temperature $\boldsymbol{T} ; 2$ ) concentration of impurity $\boldsymbol{C} ; 3$ ) internal heat $\boldsymbol{H}$ which is taken into account at the modeling phase transitions and determines the ratio of impurity concentration in the liquid and solid phases.

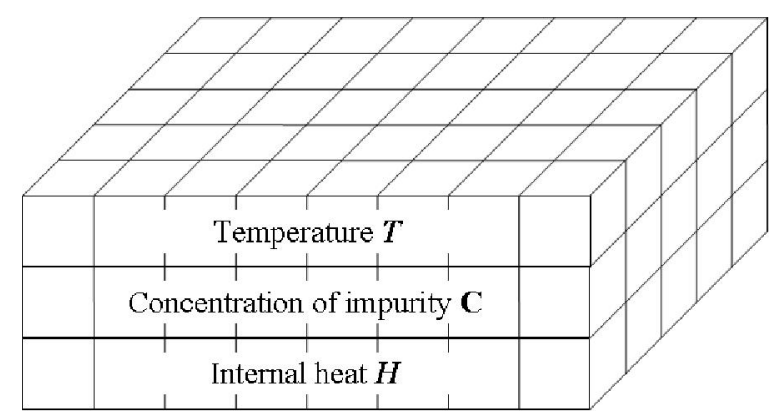

Fig. 2 - Structure of cellular automata field for a two-dimensional model. 
The modeling process is an iterative cycle of cellular automata interactions. Here we use asynchronous scheme of cellular automata interactions. This scheme involves the cyclical performance consisting of three typical steps:

1. In the cell-automaton field a cell $i=1$ with integer coordinates $x^{l}, y^{I}$ was randomly selected. Thus all cells are equiprobable for their choice.

2. A neighboring cell $i=2$ with integer coordinates $x^{2}, y^{2}$ is selected in a random equiprobable way. As neighborhood schemes is entered a Neumann neighborhood in this case, i.e. the cells have only four neighbors (Fig. 3):

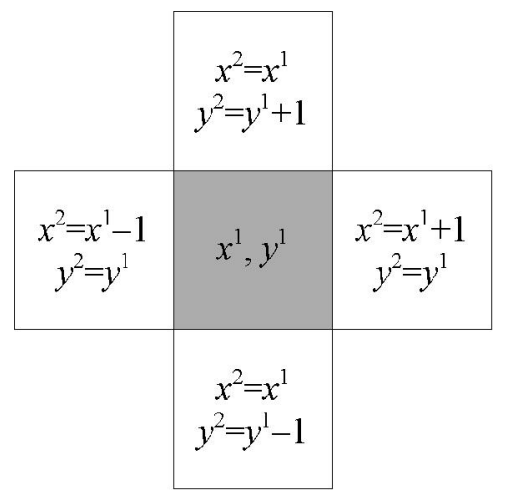

Fig. 3 - Neumann neighborhood and cell coordinates to a two-dimensional model.

3. The cellular automata interaction between the two cells takes place.

The content of the field cells can be of real continuous values.

The essence of cellular automata interactions is in modification of continuous values of the corresponding layers of cells according to the following system of equations:

$$
\begin{aligned}
& \int T^{i \prime}=T^{i}+\left(T_{\mathrm{av}}-T^{i}\right) A_{\mathrm{av}} / M_{\mathrm{max}} ; \\
& C^{i}{ }^{\prime}=C^{i}+\left(C_{\mathrm{av}}-C^{i}\right) D_{\mathrm{av}} / M_{\text {max }} ; \\
& \text { if }\left(T^{i \prime}>T_{\mathrm{m}}^{i}\right) \text { and }\left(H^{i}<H_{\mathrm{m}}\right) \\
& \text { then: }\left\{H^{i{ }^{\prime}}=H^{i}+\Delta H^{i} ; \quad T^{i{ }^{\prime}}=T_{\mathrm{m}}^{i} ;\right\} \\
& \text { if }\left(H^{i}>H_{\mathrm{m}}\right) \\
& \text { then: }\left\{\begin{array}{l}
T^{i{ }^{\prime}}=T_{\mathrm{m}}^{i}+\left(H^{i \prime}-H_{\mathrm{m}}\right) / q_{L}^{i} ; \\
H^{i \prime}=H_{\mathrm{m}} ;
\end{array}\right\} \\
& \operatorname{if}\left(T^{i{ }^{\prime}}<T_{\mathrm{m}}^{i}\right) \text { and }\left(H^{i}>0\right) \\
& \text { then: }\left\{H^{i{ }^{\prime}}=H^{i}+\Delta H^{i} ; \quad T^{i{ }^{\prime}}=T_{\mathrm{m}}^{i} ;\right\} \\
& \text { if }\left(H^{i \prime}<0\right) \\
& \text { then: }\left\{T^{i{ }^{\prime}}=T_{\mathrm{m}}^{i}+H^{i{ }^{\prime}} / q_{S}^{i} ; \quad H^{i{ }^{\prime}}=0 ;\right\}
\end{aligned}
$$

Here $i=1,2-$ index corresponding to the selected and adjacent cells respectively; $a v$ - average value index; a stroke was used to mark values at the next moment in time. $T$ - temperature, $A-$ coefficient of temperature conductivity, $\eta-$ coefficient of heat conductivity, $q$ - specific heat, $\rho-$ specific density, $C-$ concentration of impurities, $D$ - diffusion coefficient of impurities, $H$ 
- internal heat. The parameters for the solid and liquid phases are marked with lower indices $S$ and $L$ respectively. Also: $H_{\mathrm{m}}$ - latent heat of melting, $T_{\mathrm{m}}(0)$ - melting point at zero concentration of impurities, $K_{0}$ - equilibrium coefficient of segregation of impurities, $\operatorname{tg}(\alpha)$ - tangent of the angle of incline of the concentration dependence of the melting temperature (curve Solidus).

The boundary conditions are selected as follows: on the one side sample is heated, on the other cooled, the top and the bottom of the sample are thermoisolated.

In our case of modeling segregation phenomena the basic idea lies in linking impurity concentration of solid and liquid phases to corresponding ratio of internal heat and to latent heat of melting. It is schematically illustrated by Fig. 4 .

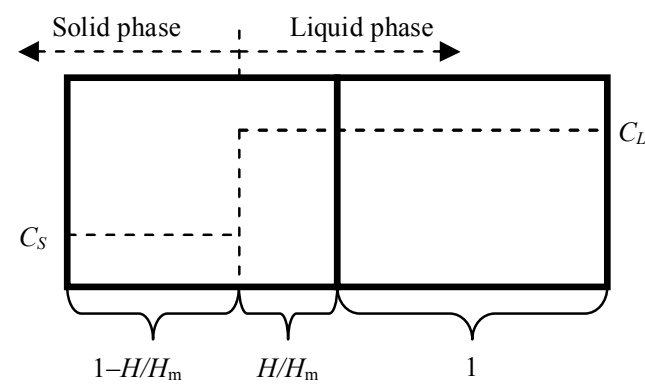

Fig. 4-Schematic representation of the process of determining impurity concentration in a cell in the state of melting or crystallization during interaction with the neighbouring cell.

As it is suggested in $[16,19]$ the simulation time of one cellular automata interaction depends on the dimensions of the field as well as the maximum coefficient of thermal conductivity. However in our case, another mechanism of diffusion of impurities is added so a certain parameter $M_{\max }$ and it should be introduced into formulas for determining the time of one interaction:

$$
M_{\max }=\left\{\begin{array}{lll}
A_{\max }, & \text { if } & A_{\max }>D_{\max } \\
D_{\max }, & \text { if } & D_{\max }>A_{\max }
\end{array} .\right.
$$

This parameter is also included into the system of equations (3).

It is clear that to ensure the adequacy of the modeling process of cellular automata the field dimension should be as large as possible. On the other hand, it will inevitably lead to a rather long modeling process. The problem of finding a compromise between the solution accuracy and the time needed to obtain it arises here, similarly to many other decision schemes. According to [16], a range of automatic single-cell interaction depends on the dimension of the field.

For a one-dimensional model, which has a field of $N_{x}$ cells the time of interaction is:

$$
t_{1 C A}=\frac{d_{x}^{2}}{a_{\max }} \frac{1}{2 N_{x}^{3}} .
$$

For a two-dimensional model $\left(N_{x} \times N_{y}\right.$ cells $)$ :

$$
t_{1 C A}=\frac{d_{x}^{2}}{a_{\max }} \frac{1}{4 N_{x}^{3} N_{y}}=\frac{d_{y}^{2}}{a_{\max }} \frac{1}{4 N_{y}^{3} N_{x}} .
$$

For a three-dimensional model $\left(N_{x} \times N_{y} \times N_{z}\right.$ cells):

$$
\begin{gathered}
t_{1 C A}=\frac{d_{x}^{2}}{a_{\max }} \frac{1}{6 N_{x}^{3} N_{y} N_{z}}=\frac{d_{y}^{2}}{a_{\max }} \frac{1}{6 N_{y}^{3} N_{x} N_{z}}= \\
=\frac{d_{z}^{2}}{a_{\max }} \frac{1}{6 N_{z}^{3} N_{y} N_{x}}
\end{gathered}
$$

here $d_{x}, d_{y}$ and $d_{z}$ - the dimensions of the sample along the coordinates $x, y$ and $z$ respectively.

Thus, the larger dimension of the CA field, the greater the number of cellular automata interactions should hold the model during a certain period of time. On the other hand, according to [20], this also increases the accuracy of calculations.

\section{SOFTWARE IMPLEMENTATION OF CA MODEL}

Possibilities of TMT Pascal have been used to write a program that implements the interaction of cellular automata.

The initial values of temperature, impurity concentration and internal heat for each point (cell of cellular-automata field) of the sample have been set using three-dimensional array.

Automata asynchronous communication carried considering above described boundary conditions of the process. To calculate the new states entered the cycle. A cell from Neumann neighbourhood equiprobable selected for a random cell at each iteration and its new state was calculated. That is, the calculation of temperature, impurity concentration and internal heat at the next moment was carried out according to the equation system (3). Afterwards again next two adjacent cells are randomly selected and parameters are calculated. Such cyclical repetition of these steps provides for determining the values of all three fields of the sample at any given time. The flowchart of the computation algorithm is shown in Fig. 5. It is not applicable text output information. They use 
matching color gradation with the value of the displayed parameter to facilitate the program listings understanding.

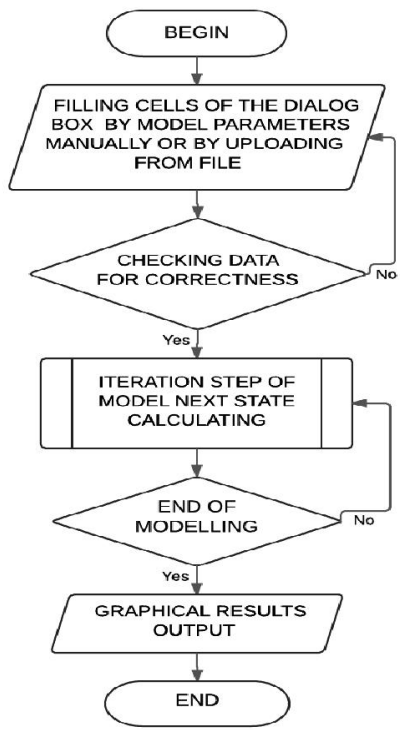

Fig. 5 - Flowchart of the developed algorithm of CA modeling

Thus visualized process can be seen on the screen in dynamics. Computer with following parameters was used for computational experiments: CPU AMD K-7, RAM - 1.5 GB; HDD - 40 GB; OS Windows XP., a number of cellular automata interactions was fixed during the experiments. The averaged values of the number of interactions at different time moments $t$ and with different number of cellular-automata field cells $\mathrm{N}$ are listed in Table 1.

Table 1. Results of computational experiments

\begin{tabular}{|l|l|l|l|l|l|}
\hline \begin{tabular}{l}
\hline $\mathbf{N}$ \\
$t$
\end{tabular} & 0,001 & 0,002 & 0,003 & 0,004 & 0,005 \\
\hline 100 & 2000 & 4000 & 6000 & 8000 & 10000 \\
\hline 200 & 16000 & 32000 & 48000 & 64000 & 80000 \\
\hline 300 & 54000 & 108000 & 162000 & 216000 & 270000 \\
\hline 400 & 128000 & 256000 & 384000 & 512000 & 640000 \\
\hline 500 & 250000 & 500000 & 750000 & 1000000 & 250000 \\
\hline
\end{tabular}

The computation time is highly dependent on the fact how often (every interaction or, for example, 1 in a million CA-interactions) the result is displayed on the screen. For example, 250000000 interactions with output of each result on the screen requires 10 seconds, if not display - 10 times faster (about 1 second).Therefore, the ideal environment for the CA models implementation are highly parallel systems.

\section{CALCULATION RESULTS AND ANALYSIS}

It is clear that a model that does not reproduce the full process it describes should not be used.
Therefore, to confirm the possibility of cellular automata modeling of heat conduction process a series of numerical experiments was conducted.

The temperature distribution dynamics in the sample with a "step" initial condition is described by the following law for the one-dimensional case:

$$
p(x, t)=\frac{T_{0}}{2 \sqrt{\pi a t}} \exp \left(-\frac{x^{2}}{4 a t}\right) .
$$

Let's shift the initial point of abrupt temperature distribution in the middle of the unit interval and integrate expression (8) to obtain temperature distribution:

$$
T(x, t)=\int_{-\infty}^{x} p(z-0.5, t) d z
$$

In the computational experiments the family of distributions for temperatures in diapason $x \in[0,1]$ at different times was constructed [21].

Fig. 6 shows the results of calculations by formulas (8) and (9) in comparison with the above described continuous asynchronous cellular automata method.

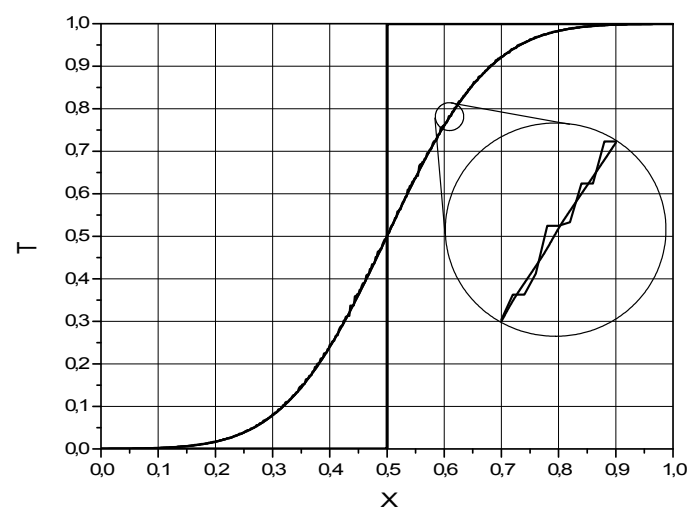

Fig. 6 - Temperature distribution in the sample at time $t=0,01 \mathrm{~s}(N=500)$.

Smooth line - the decision by the formulas (8) and (9), broken - cell-automaton solutions.

At the right part of the figure - tenfold increased temperature distribution fragment.

The high degree of coincidence confirms the adequacy of the use of cellular automata approach to approximate solutions of non-stationary heat equation.

Let's try to apply CA model on thermal conductivity process simulation with the first-order phase transition [16] on the example of bismuth telluride $\left(\mathrm{Bi}_{2} \mathrm{Te}_{3}\right)$ melting zone. Practically, the problem of modeling the growing process of semiconductor materials zone is important, in 
particular of $\mathrm{Bi}_{2} \mathrm{Te}_{3}$ that is used in various thermoelectric devices (Fig. 7).

$\mathrm{Bi}_{2} \mathrm{Te}_{3}$ thermal parameters are presented in Table 2, and the process parameters include the heater temperature $-840{ }^{\circ} \mathrm{C}$, refrigerator temperature $-30{ }^{\circ} \mathrm{C}$, the speed of growth (speed of heaters movement) $-200 \mathrm{~mm} / \mathrm{h}$, the height of the fragment vials $-15 \mathrm{~cm}$, internal thickness of ampoules $-3 \mathrm{~cm}$, height heater $-5 \mathrm{~cm}$. The movement of the heater is upward (from the bottom to the top). At the bottom of the ampoule a single crystal is formed, and molten zone flows as a polycrystal at the top. The sizes of CA fields $N_{x} \times N_{y}=120 \times 600$ cells.

Table 2. $\mathrm{Bi}_{2} \mathrm{Te}_{3}$ thermal parameters.

\begin{tabular}{|c|c|c|c|}
\hline & 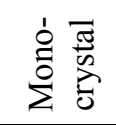 & $\begin{array}{l}\frac{\pi}{0} \\
0 \\
0\end{array}$ & $\sum_{\Sigma}^{ \pm}$ \\
\hline $\begin{array}{l}\text { Coefficient of thermal } \\
\text { conductivity, } \mathrm{W} /\left(\mathrm{m} \times{ }^{\circ} \mathrm{C}\right)\end{array}$ & 2 & 3 & 5 \\
\hline Density, $\mathrm{kg} / \mathrm{m}^{3}$ & 7700 & 7600 & 7400 \\
\hline Specific heat, $\mathrm{J} /\left(\mathrm{kg} \times{ }^{\circ} \mathrm{C}\right)$ & 300 & 300 & 330 \\
\hline Melting point, ${ }^{\circ} \mathrm{C}$ & 585 & 585 & 585 \\
\hline $\begin{array}{l}\text { The heat of mel- } \\
\text { ting/crystallization, } \mathrm{J} / \mathrm{kg}\end{array}$ & 300000 & 300000 & 300000 \\
\hline
\end{tabular}
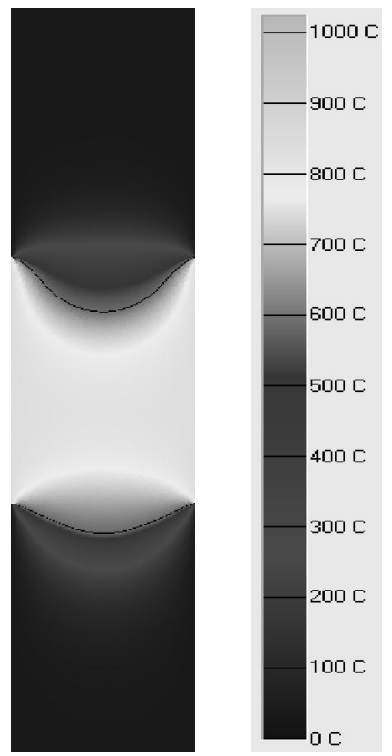

Fig. 7 - Example of $\mathrm{Bi}_{2} \mathrm{Te}_{3}$ zone growing process simulation.

It is possible to observe various forms of crystallization front - convex toward the melt, concave or flat by changing the parameters of $\mathrm{Bi}_{2} \mathrm{Te}_{3}$ zone growing. The parameters of growing in $\mathrm{CA}$ simulation, in which there is a flat crystallization front is the same as the experimental ones.

Taking into account the impurity subsystem (3) it is possible to model the segregation phenomenon.
A series of computational experiments was conducted to confirm the adequacy of the proposed model. An example is presented in Fig. 8.

The given results of calculations of distribution of the relative impurity concentration along the sample fragment during crystallization are quite consistent with experimental data.
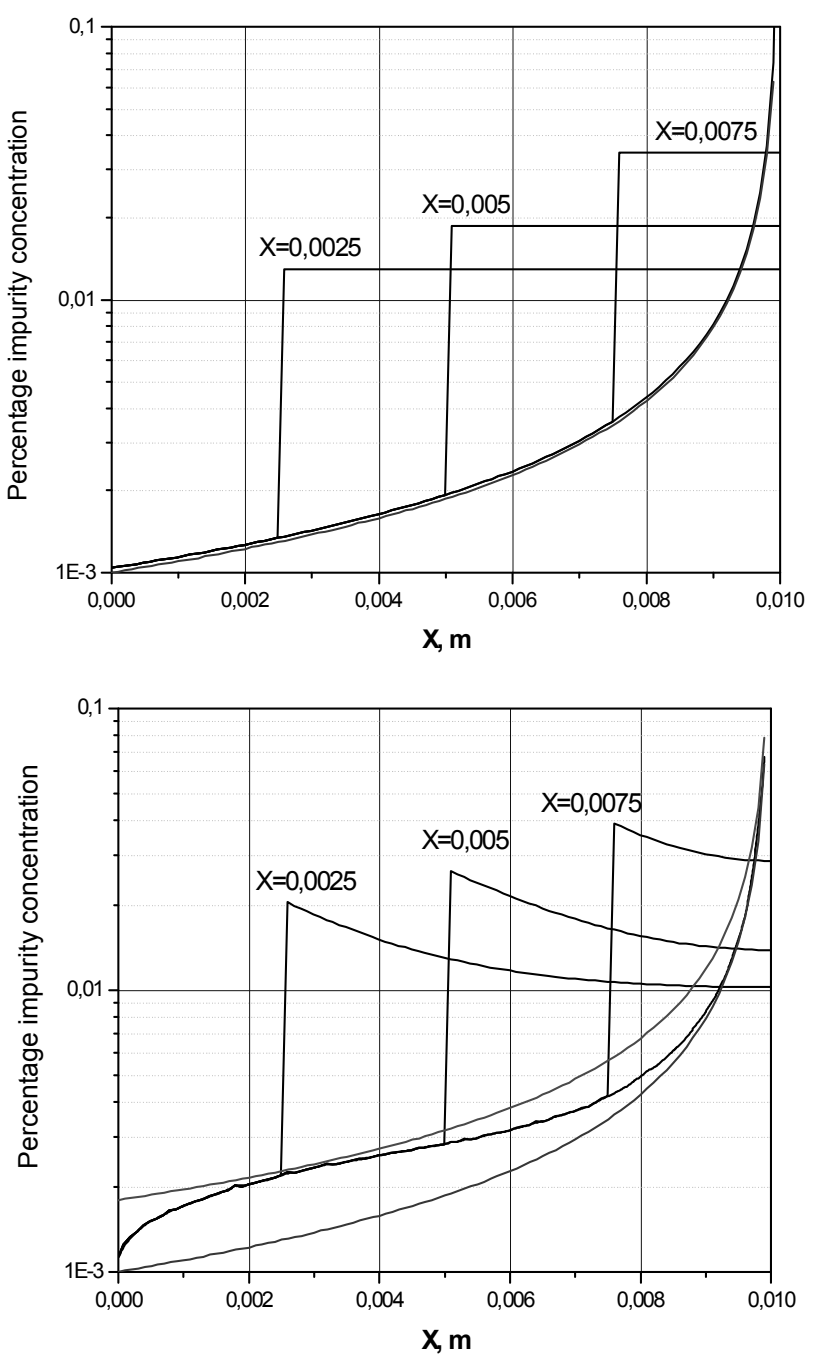

Fig. 8 - Distribution of the relative concentration of impurities along the sample fragment during crystallization (the intermediate results of crystallization front movement are shown at three points).

The lower curve (Fig. 8) presents the equilibrium crystallization under conditions of infinitely low growth velocity. For the top value the growth velocity $V=0,1 \mathrm{~mm} / \mathrm{h}$, for the lower one $V=30$ $\mathrm{mm} / \mathrm{h}$. The diffusion coefficient of impurities in the liquid phase $D_{L}=10^{-8} \mathrm{~m}^{2} / \mathrm{s}$. The segregation coefficient $K_{0}=0,1$. The higher curve - equilibrium crystallization with segregation coefficient $K_{0}=0,8$.

The equation that describes the distribution of impurities at directional crystallization has the following form [17]: 


$$
\frac{d x}{L}=\frac{d C_{L}}{C_{S}-C_{0}}
$$

It is obvious from the first graph of distribution of relative concentration of impurities (Fig. 8) that under a very slow increase of the velocity, the distribution calculated according to (3) practically coincides with the analytical solution of the equation (10):

$$
C_{S}=K C_{0}\left(1-\frac{x}{L}\right)^{k-1},
$$

where $K$ - coefficient of segregation of impurities, $C_{0}$ - initial concentration of impurities, $L-$ length of the sample.

While increasing the velocity (the lower graph of Fig. 8), a deviation from dependence (11) is observed. This deviation can be explained taking into account the effective distribution coefficient, not the equilibrium one. By analyzing formula (2) which unites efficient and equilibrium distribution coefficients, we can conclude that the diffusion layer thickness $\delta$ is not constant and varies in the range of $1 \mathrm{~mm}$. This is quite consistent with the corresponding value for impurities with the diffusion coefficient in the liquid phase $D_{L}=10^{-8} \mathrm{~m}^{2} / \mathrm{s}$ [18].

The adequacy of the cellular automata model (3) was also tested on modeling of the phenomenon of concentration overcooling while growing doped crystals. Here is a brief description of this phenomenon.

According to the phase diagram (Fig. 1), each point of the melt will have its corresponding liquidus temperature. Let $K_{0}<1$, then the content of impurities in the melt adjacent to the division boundary increases and the equilibrium liquidus temperature in this area should decrease.

A temperature gradient is observed in the melt. It is created to provide the crystal growth process. If the temperature of the melt at some distance from the division surface is below the liquidus temperature, the melt will be overcooled. Overcooling will take place despite the fact that the temperature of the melt is higher than that of the phase division surface.

This creates a situation where the melt is heated more than the crystal is, but still is overcooled. A crystal can now serve as a starter that causes further crystallization of the overcooled melt trying to eliminate overcooling. There is also a probability of appearance of such a starter of crystallization in the depth of the melt near the border of the phase division. The greater the degree of overcooling, the greater the probability of appearance of such a starter.

The existence of such an overcooling was initially observed by Rutter and Chalmers in 1953 [21]. They called this overcooling a "concentration overcooling" emphasizing that it occurs as a result of change of impurity concentration in the melt.

During crystallization of the melt with no impurities or the melt with impurities at vigorous stirring, only a thermal overcooling takes place. Concentration overcooling occurs due to insufficient stirring when the boundary layer of the melt is enriched (or depleted at $K_{0}>1$ ) by impurities. The concentration overcooling leads to the formation of cellular structure that violates the uniformity of a monocrystal, and is therefore undesirable.

Quantitative description of the directed crystallization of the binary melt using a fairly simple model [21] leads to an important result for the practice of crystal growth from the melt - a criterion of concentration overcooling. The essence of this criterion can be expressed through the critical speed of crystallization [21]:

$$
V_{\text {crit }}=\frac{G D_{L}}{\left(K_{0}-1\right) \operatorname{tg}(\alpha) C_{0}}
$$

where $G$ - the temperature gradient in the front of crystallization directed towards the melt; $C_{0}$ - initial relative concentration of impurities.

Undesirable overcooling of the melt such as concentration overcooling can be avoided if a certain limit of crystallisation velocity $\left(V_{\text {crit }}\right)$ is not exceeded. This limit can be raised by either increasing the temperature gradient at the phase boundary $(G)$ or lowering the content of impurities in the starting material $\left(C_{0}\right)$. The cellular crystal growth can be avoided if this rule is followed.

Fig. 9 presents the results of calculations of the relative concentration of impurities (top graph) and temperature distribution (bottom graph) along a sample.

The main process parameters: $G=2{ }^{\circ} \mathrm{C} / \mathrm{cm} ; D_{L}=$ $10^{-7} \mathrm{~m}^{2} / \mathrm{c} ; V_{\text {crit }}=30 \mathrm{~mm} / \mathrm{h} ; V=100 \mathrm{~mm} / \mathrm{h}$.

Fig. 8 shows that exceeding of a certain critical value of the speed growth leads to unstable behavior of the crystallization front, and thus - to an uneven distribution of impurities in the solid phase.

The parameters of tin-antimony (Sn-Sb) [22] were chosen as thermodynamic parameters of the material, where tin is the basic material and antimony is an admixture with relative concentration $C_{0}=0.01$, i.e. $1 \%$. The main thermal parameters for 
tin in solid and liquid phases are [23]: coefficient of thermal conductivity $\eta_{S}=65,8 \mathrm{~W} / \mathrm{m}^{\circ} \mathrm{C} ; \eta_{L}=30,5$ $\mathrm{W} / \mathrm{m}^{\circ} \mathrm{C}$; specific heat $q_{S}=226 \mathrm{~J} / \mathrm{kg}^{\circ} \mathrm{C} ; q_{L}=268$ $\mathrm{J} / \mathrm{kg}^{\circ} \mathrm{C}$; specific density $\rho_{S}=7300 \mathrm{~kg} / \mathrm{m}^{3} ; \rho_{L}=$ $6980 \mathrm{~kg} / \mathrm{m}^{3}$; latent heat of melting $H_{\mathrm{m}}=60.7 \mathrm{~kJ} / \mathrm{kg}$; melting point at zero concentration of impurities $T_{m}(0)=232^{\circ} \mathrm{C}$; equilibrium segregation coefficient of the impurity ( $\mathrm{Sb}$ ) $K_{0}=10$; tangent of the angle of incline of the concentration dependence of the melting temperature (curve Solidus) $\operatorname{tg}(\alpha)=25$.
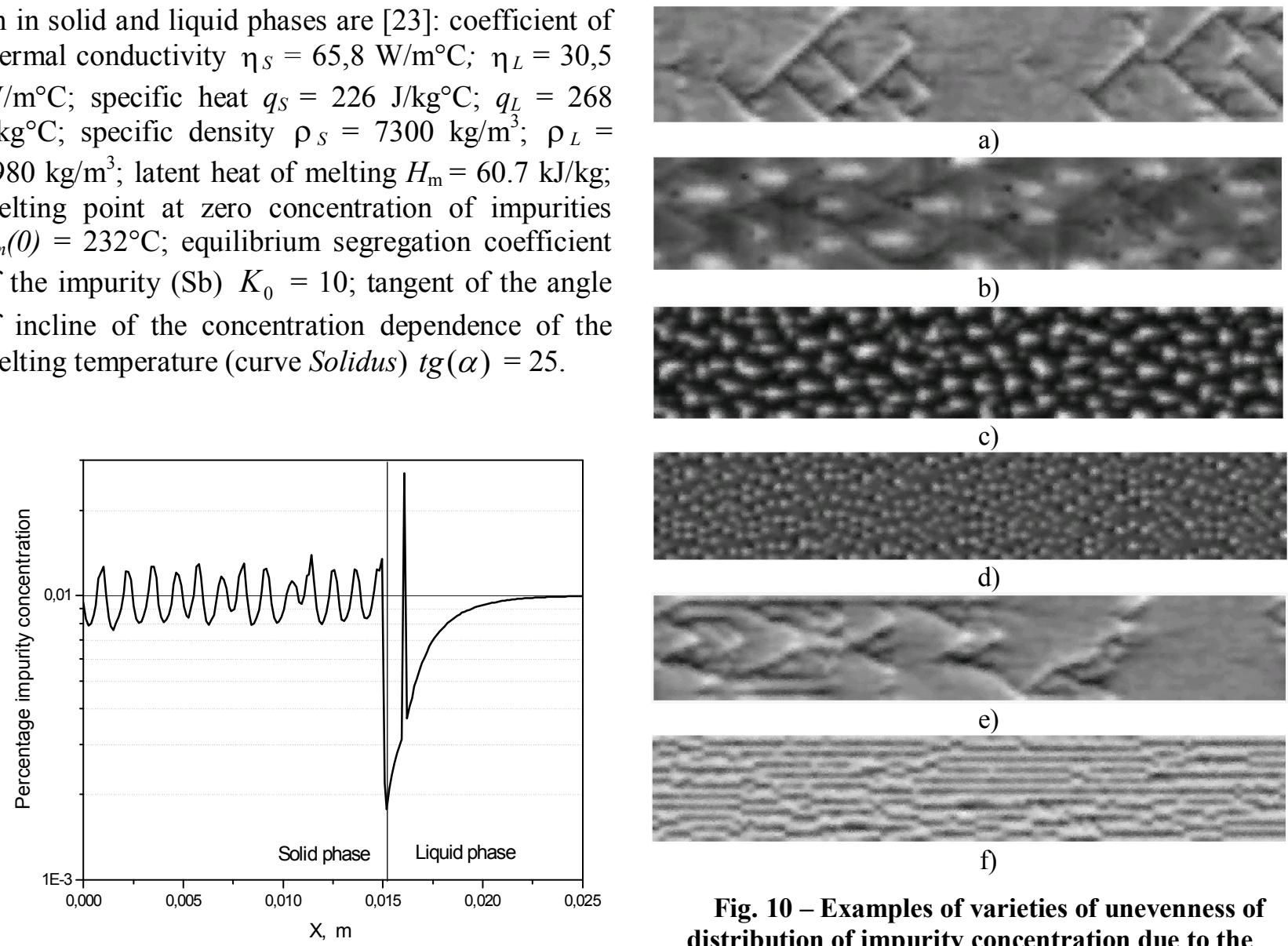

a)

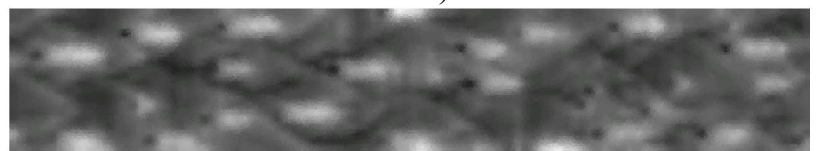

b)

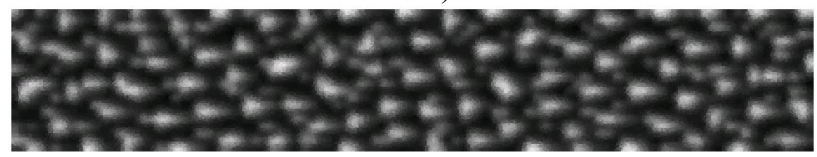

c)

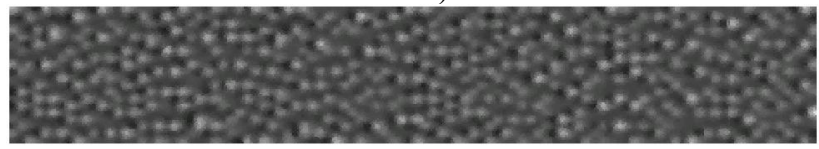

d)

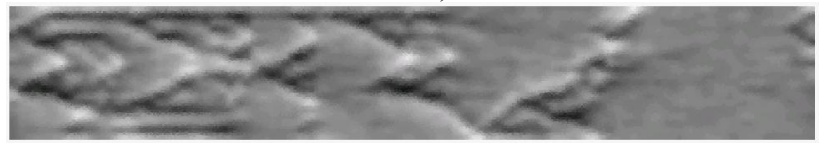

e)

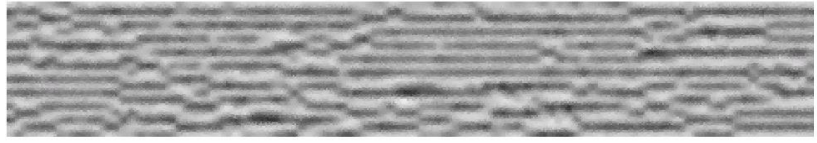

f)

Fig. 10 - Examples of varieties of unevenness of distribution of impurity concentration due to the concentration overcooling (cellular crystal growth).

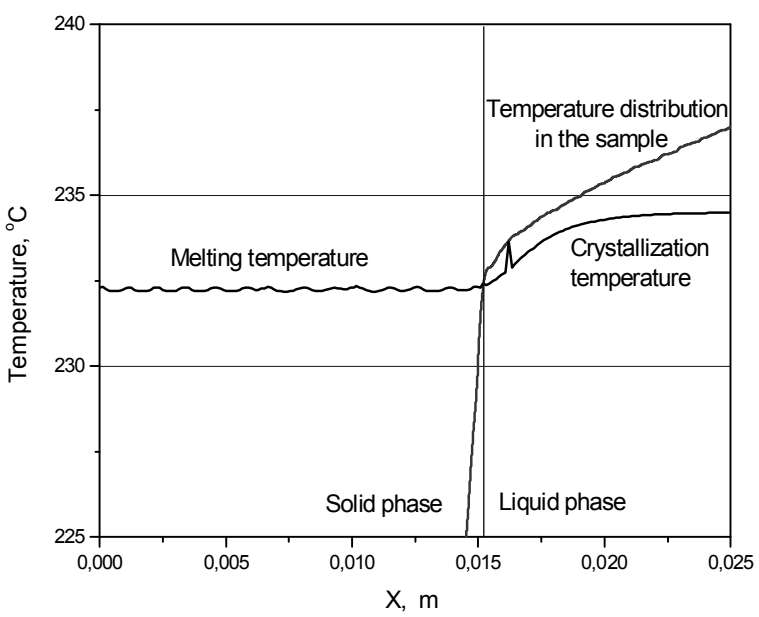

Fig. 9 - Distribution of the relative concentration of impurities (top graph) and temperature in a sample, as well as temperature of melting and crystallization (lower graph).

The results of modeling distribution of impurity concentration in two- or three-dimensional cases are rather interesting. The variety of cellular structures can be seen in Fig. 10.

The main model parameters that led to corresponding characteristic distribution of impurity concentration (Fig. 10) are listed in Table 3.

Table 3. The main model parameters.

\begin{tabular}{|c|l|l|l|l|l|l|}
\hline Sample: & a) & b) & c) & d) & e) & f) \\
\hline$G,{ }^{\circ} \mathrm{C} / \mathrm{cm}$ & 8 & 2 & 2 & 0,3 & 25 & 6 \\
\hline$D_{L}, \mathrm{~m}^{2} / \mathrm{s}$ & $10^{-7}$ & $10^{-7}$ & $10^{-8}$ & $10^{-8}$ & $10^{-8}$ & $10^{-8}$ \\
\hline$V_{\text {crit }}, \mathrm{mm} / \mathrm{h}$ & 125 & 30 & 2,5 & 0,5 & 40 & 10 \\
\hline$V, \mathrm{~mm} / \mathrm{h}$ & 100 & 100 & 100 & 100 & 30 & 30 \\
\hline$C_{\min }$ & $\begin{array}{c}89 \times \\
\times 10^{-4}\end{array}$ & $\begin{array}{c}74 \times \\
\times 10^{-4}\end{array}$ & $\begin{array}{c}19 \times \\
\times 10^{-4}\end{array}$ & $\begin{array}{c}28 \times \\
\times 10^{-4}\end{array}$ & $\begin{array}{c}86 \times \\
\times 10^{-4}\end{array}$ & $\begin{array}{c}72 \times \\
\times 10^{-4}\end{array}$ \\
\hline$C_{\max }$ & $\begin{array}{c}111 \times \\
\times 10^{-4}\end{array}$ & $\begin{array}{l}143 \times \\
\times 10^{-4}\end{array}$ & $\begin{array}{l}325 \times \\
\times 10^{-4}\end{array}$ & $\begin{array}{c}259 \times \\
\times 10^{-4}\end{array}$ & $\begin{array}{l}114 \times \\
\times 10^{-4}\end{array}$ & $\begin{array}{l}117 \times \\
\times 10^{-4}\end{array}$ \\
\hline
\end{tabular}

It is clear from the analysis of Table 3 and Figure 9 that the occurrence of concentration overcooling in the process of crystal growth is consistent with the estimated equation (12). The more the actual speed $V$ exceeds the critical limit $V_{\text {crit }}$, the more pronounced the phenomenon of cellular growth is. Fig. 11.a, b shows the examples of the varieties of shapes of crystallization front under concentration overcooling. Solid phase is shown on the left side of the figure and the liquid phase is shown on the right. Dark areas correspond to the minimum value of the impurity concentration, light areas - to the maximum one. It can be seen that both the 
appearance of starters of crystallization in depth of the melt near the border of the phase division (Fig. 10.a) and cellular growth of the solid phase exceptionally from the surface of the monolithic crystal (Fig. 11.b) are possible. Fig. 11.c shows flat crystallization front and the lack of uneven distribution of impurities in the solid phase. This may occur at low speeds and high crystallization front temperature gradient in the front of crystallization. Otherwise there a phenomenon of concentration supercooling will occur. The search for optimal conditions, i.e. maximum speed and lowest temperature gradient when the concentration supercooling will not occur (Fig. 11.c) is the main economic challenge.

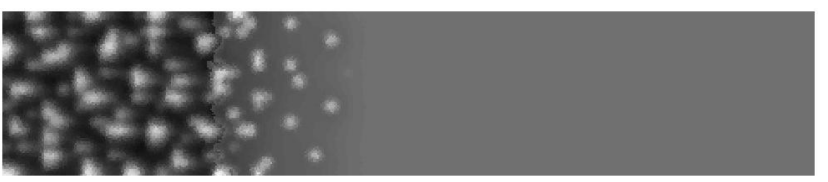

a) $C_{\min }=0,0009 ; C_{\max }=0,068$

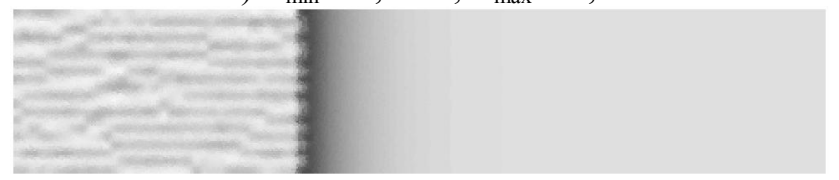

b) $C_{\min }=0,0014 ; C_{\max }=0,012$.

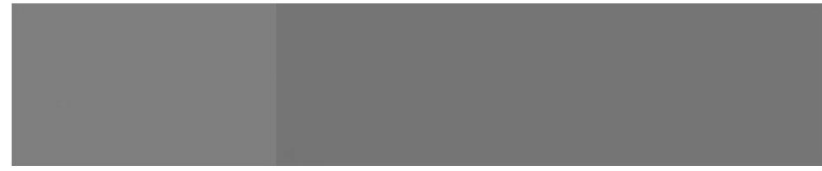

c) $C_{\min }=0,0098 ; C_{\max }=0,0101$.

Fig. 11 - Examples of varieties of forms of the crystallization front under concentration overcooling (cellular crystal growth).

Fig. 11 (a) is consistent with the results shown in Fig. 10.c, while Fig. 11 (b) - with those in Fig. 10.f.

\section{CONCLUSIONS}

Thus, mathematical modeling of dynamic processes is the most frequent and developed direction of application of cellular automata. It is often occurs the situation when considering problem can not be solved analytically, and the calculation of it as the difference scheme gives rise to various kinds of instabilities while mathematical modeling of physical phenomena. A number of problems arise when solving problems in areas with a complex shape.

Replacement of the physical reality, often wearing a discrete character, by the continuous model occurs in the process of describing the physical phenomenon with the help of set of differential equations. Space and time in the continuous model made again the discrete while transition back to difference schemes and all values are considered with the limited accuracy after implementing them on the computer.

Hence we can conclude that it is advisable to immediately build digital models of physical phenomena, which are cellular automata.

This research work sets out the detailed methodology of modeling the phenomenon of impurities segregation in crystalline materials by means of continuous cellular automata.

As a result of a series of computational experiments, the feasibility of the proposed model was confirmed.

The article presents the results of calculations of the relative concentration of impurities and temperature distribution along the sample.

A well-known criterion for the occurrence of concentration overcooling was used for the comparative analysis of the adequacy of the obtained results. It is the critical speed of crystallization (12) that depends on the temperature gradient at the boundary of phase division and on the initial relative concentration of the impurity. Changes of these parameters lead to the changes in critical speed and thus enable to avoid such an undesirable effect as cellular growth of the crystal.

The suggested model, apart from attaching the moment of transition to the concentration overcooling, enables to determine the nature of cellular growth.

However, the known cellular automata are not fast enough for the simulation of problems of inhomogeneous dynamic systems in large scale and on detailed lattices. Therefore, improvement of the proposed numerical method is very promising.

\section{REFERENCES}

[1] J. von Neumann, Theory of Self-reproducing Automata, edited by A. Burks, Univ. of Illinois Press, 1966.

[2] W. Li, N. H. Packard, C. G. Langton, Transition Phenomena in Cellular Automata Rule Space, Physica D, (45) (1990), pp. 77-94.

[3] S. Wolfram, A New Kind of Science, Champaign, IL: Wolfram Media, 2002.

[4] H. Chate and P. Manneville, Criticality in cellular automata, Physica D, (45) (1990), pp. 122-135.

[5] H. Gutowitz, A Hierarchical Classification of Cellular Automata, Physica D, (45) (1990), pp. 136-156.

[6] H. McIntosh, Wolfram's class IV automata and a good Life, Physica D, (45) (1990), pp. 105121.

[7] O.L. Bandman, Cellular Automata models of spatial dynamics, System Informatics, (10) (2005), pp. 57-113. 
[8] V.K. Vanag, Study of spatially extended dynamical systems using probabilistic cellular automaton, Physical Sciences Progress, (5) 169 (1999), pp. 481-505.

[9] G. Malinetskij, M.E. Stepantsov, Simulation of diffusion processes cellular automata with Margoles neighbourhood, Computational Mathematics and Mathematical Physics, (6) 36 (1998), pp. 1017-1021.

[10] S. Bobkov, Y.V. Voytko, Usage of cellular automata to model the nonlinear heat conduction problems, Chemistry and Chemical Engineering, (11) 52 (2009), pp. 126-128.

[11] N. Limanova, E. Mamzin, S. Matveev, Modeling of heat transfer, Bulletin of the Samara State Aerospace University, (19) 3 (2009), pp. 265-269.

[12] J. M. Benito and P. Hernández, Modeling segregation through cellular automata: a theoretical answer, WP-AD, (2007), 16.

[13] T. C. Schelling, Dynamic Models of Segregation, Journal of Mathematical Sociology, (1) 2 (1971), pp. 143-186.

[14] K. G. F. Janssens, An introductory review of cellular automata modeling of moving grain boundaries in polycrystalline materials, Mathematics and Computers in Simulation, (80) 7 (2010), pp. 1361-1381.

[15] R. Golab, D. Bachniak, K. Bzowski, L. Madej, Sensivity Analysis of the Cellular Automata Model for Austenite-Ferrite Phase Transformation in Steels, Applied Mathematics, (4) (2013), pp. 1531-1536.

[16] V.V. Zhikharevich, L.M. Shumylyak, L.T. Strutinskaya, S.E. Ostapov, Construction and investigation of continuous cellular automaton model of the processes of heat conduction with the first order phase transitions, Computer Studies and Modeling, (5) 2 (2013), pp. 141-152.

[17] J.K. Yezhovski, O.V. Denisova, Physical and Chemical Bases of Technology of Semiconductor Materials: Proc. Allowance, $\mathrm{SPb}$ : : SZTU, 2005.

[18] J.A. Burton, R.C. Prim, W.P. Slichter, The Distribution of Solute in Crystals Growth from the Melt. Part I. Theoretical, J. Chem. Phys., (21) 11 (1953), pp. 1987-1991.

[19] L. T. Strutynska, V.V. Zhiharevich, Computer simulation of the conditions of formation of flat crystallization front in the growing of thermoelectric material, Physics and Chemistry of Solids, (13) 40 (2012), pp. 1041-1046.

[20] V.V. Zhikharevich, L.M. Shumylyak, Approximation of the solution of the nonstationary equation of heat conductivity by the method of probabilistic continuous asynchronous cellular automats for a onedimensional case, Computer Studies and Modeling, (4) 2 (2012), pp. 293-301.

[21] W.A. Tiller, J.W. Rutter, K A. Jackson, B. Chalmers, The redistribution of solute atoms during the solidification of metals, Acta Metallurgica, (8) 4 (1953), pp. 428-437.

[22] N. P. Lyakisheva, The state diagram of binary metal systems, Mechanical Engineering, (1996-2000).

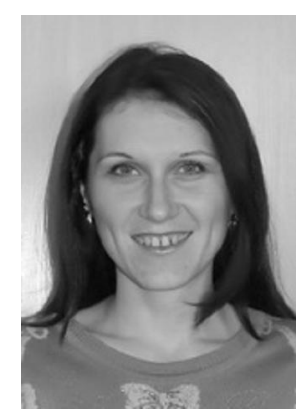

Lilia Shumilyak, Master of Physical and Biomedical Electronics, Assistant of Department of Information Systems, Chernnivtsi Faculty of National Technical University "Kharkiv Politechnical Institute".

Research interests: Cellular automata modeling.

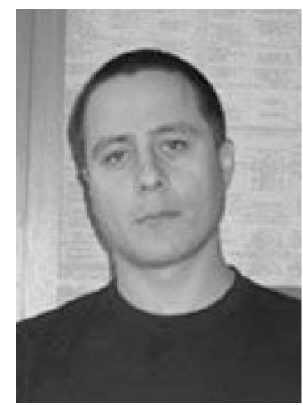

Vladimir Zhikharevich, PhD. of physical and mathematical sciences, associate professor of the Department of Computer Systems Software, Yuriy Fedkovych Chernivtsi National University.

Research interests: Application problems in the theory of cellular automata, the theory of self-organizing systems (synergy).

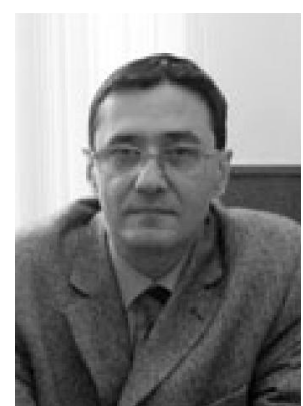

Sergey Ostapov, Dr. Sci., Professor Ostapov S. E., head of the Department of Computer Systems Software, Yuriy Fedkovych Chernivtsi National University.

Research interests: Cellular automata modeling, intelligent analysis and processing of information, components of artificial intelligence in engineering tasks. 